\title{
Interference of Resonance Fluorescence from two four-level atoms
}

\author{
T. Wong, S.M. Tan, M.J. Collett and D.F. Walls \\ Department of Physics, \\ University of Auckland, Private Bag 92019, Auckland, New Zealand
}

\begin{abstract}
In a recent experiment by Eichmann et al., polarization-sensitive measurements of the fluorescence from two four-level ions driven by a linearly polarized laser were made. Depending on the polarization chosen, different degrees of interference were observed. We carry out a theoretical and numerical study of this system, showing that the results can largely be understood by treating the atoms as independent radiators which are synchronized by the phase of the incident laser field. The interference and its loss may be described in terms of the difference between coherent and incoherent driving of the various atomic transitions in the steady-state. In the numerical simulations, which are carried out using the Monte Carlo wave function method, we remove the assumption that the atoms radiate independently and consider the photodetection process in detail. This allows us to see the total interference pattern build up from individual photodetections and also to see the effects of superfluorescence, which become important when the atomic separation is comparable to an optical wavelength. The results of the calculations are compared with the experiment. We also carry out simulations in the non steady-state regime and discuss the relationship between the visibility of the interference pattern and which-path considerations.
\end{abstract}

PACS numbers: $32.50 .+\mathrm{d}, 03.65 . \mathrm{Bz}, 42.50 .-\mathrm{p}$

\section{INTRODUCTION}

Young's two slit experiment is the canonical experiment demonstrating the wave nature of light. With the addition of a measuring device to determine which slit the photon has passed through, the phenomenon of wave-particle duality can be investigated. Many such path detection schemes have been proposed in the past, such as the Einstein recoiling slit [1] and Feynman's light microscope [2].

Recently, interest has arisen in the interference of the fluorescent light from two driven atoms which play the role of the slits in Young's experiment. Various situations have been theoretically studied, using two-level and four-level atoms, and the possibility of placing the atoms in a cavity has also been considered. 3 . 5 ]

In a recent experiment performed by Eichmann et al. [6], the light scattered by two ${ }^{198} \mathrm{Hg}^{+}$ions in a trap was observed. In this experiment, a linearly-polarized traveling wave coherent field was used to drive the ions and the scattered light was observed in two orthogonal polarizations. An interference pattern is observed in one case but not in the other. These results were explained in two ways, firstly in terms of a which-path argument based on ways in which a single photon can interact with the two atoms and secondly in terms of a theoretical analysis by Polder and Schuurmans [7] who considered coherent driving of a single four-level atom.

In this paper, we carry out an analysis which considers the presence of both atoms and stress that for the coherent steady-state excitation as used in the experiment, the which-path argument is not applicable. In sections II to V, we extend the analysis of Polder and Schuurmans to the experimental situation. In brief, the atomic dipoles are only coherently driven in a specific direction given by the polarization of the incident light field, and so interference is only expected for scattered light of that polarization. Scattered light of the orthogonal polarization is also present, since the atoms can decay spontaneously, but this is incoherent with the driving field and does not lead to interference.

Besides the above analysis which is carried out using the master equation, a Monte Carlo wave function simulation was also carried out, as is discussed in sections VI and VII. We use the method to simulate the system without resorting to the assumptions of large atomic separation and independent radiators used in the previous analytical sections. When a fluorescent photon is detected as traveling in a given direction, it may not be possible in general to determine from which atom it arose, and this leads to additional "dipole-dipole" type of terms which become important when the atomic separation is comparable to the optical wavelength. At small separations, the atoms act cooperatively giving rise to superfluorescence whereas for large separations, we smoothly recover the results for independent radiators.

In order to compare our results with the experiment, we include the effects of the classical motion of the ions within the trap (Sec. VIII). As expected, this reduces the visibility of the interference patterns, bringing the results more closely to those observed.

In the last section (Sec. IX) we consider a situation where the two atom system is re-prepared to its initial state after each individual photon has been detected. This allows us to study the transient regime of this system and we 
find that interference is present in both polarizations, although one of these disappears in the steady-state. Depending on the initial state however, the visibility of this transient pattern varies, and a which-path argument may be used in this case to explain the results.

\section{THE MODEL}

The atoms are modeled as four-level atoms (see fig. 1) interacting with a linearly polarized light field polarized along the $z$ axis and traveling along the $y$ direction. We treat the incident laser light as a classical field and the external motion of the atoms will be neglected. The separation between the atoms will be considered to be large (many wavelengths of the laser light) so that dipole-dipole interactions can be ignored. In this approximation the atoms can be considered as independent fixed sources except that their radiation is synchronized by the definite phase of the incident laser light. The atomic dipole operator $\vec{\mu}$ is the sum of atomic raising $\mu^{\uparrow}$ and lowering $\mu^{\downarrow}$ operators whose components are given by [7]

$$
\begin{aligned}
& \mu_{x}^{\downarrow}=\mu(|1\rangle\langle 4|+| 2\rangle\langle 3|) \hat{\mathbf{x}} \\
& \mu_{y}^{\downarrow}=-\imath \mu(|1\rangle\langle 4|-| 2\rangle\langle 3|) \hat{\mathbf{y}} \\
& \mu_{z}^{\downarrow}=\mu(|2\rangle\langle 4|-| 1\rangle\langle 3|) \hat{\mathbf{z}},
\end{aligned}
$$

where $\mu_{k}^{\downarrow}$ is the $k$ component of the atomic dipole, $\mu$ is the dipole matrix element, $\hat{\mathbf{x}}, \hat{\mathbf{y}}$ and $\hat{\mathbf{z}}$ are the usual Cartesian unit vectors. The atomic operator $|i\rangle\langle j|$ couples the $i$ 'th and $j$ 'th levels of the atom. The system Hamiltonian for one of the atoms in the interaction picture is

$$
\mathbf{H}_{s}=\frac{1}{2} \hbar \Delta\left(\sigma_{44}+\sigma_{33}-\sigma_{22}-\sigma_{11}\right)+\hbar \Omega\left(\sigma_{24}+\sigma_{42}-\sigma_{13}-\sigma_{31}\right),
$$

where $\Delta$ is the detuning between the atomic transition and the incident laser light. For simplicity, the ground states (and the excited states) are considered to be degenerate in energy (see fig. 1). We define the Rabi frequency $\Omega=\mu E_{o} / 2 \hbar$ where $E_{o}$ is the electric field amplitude of the incident laser and we use the notation $\sigma_{i j} \equiv|i\rangle\langle j|$. The equation of motion for the reduced density operator $\rho_{s}$ for the single atom is

$$
\frac{d \rho_{s}}{d t}=\frac{\imath}{\hbar}\left[\rho_{s}, \mathbf{H}_{s}\right]+\mathcal{L}_{\text {relax }}\left(\rho_{s}\right)
$$

where $\mathcal{L}_{\text {relax }}$ is the relaxation superoperator

$$
\begin{aligned}
\mathcal{L}_{\text {relax }}= & -\gamma\left(\sigma_{44} \rho_{s}+\rho_{s} \sigma_{44}+\sigma_{33} \rho_{s}+\rho_{s} \sigma_{33}\right) \\
& +\gamma\left(\sigma_{14} \rho_{s} \sigma_{41}+\left(\sigma_{24}-\sigma_{13}\right) \rho_{s}\left(\sigma_{42}-\sigma_{31}\right)+\sigma_{23} \rho_{s} \sigma_{32}\right),
\end{aligned}
$$

and we have assumed that both excited states have the same decay rate $\gamma$.

\section{THE OPTICAL BLOCH EQUATIONS}

There are sixteen optical Bloch equations corresponding to the sixteen atomic operators. However, due to the geometry chosen for the incident light, the sixteen equations de-couple into two sets of eight. The first set of coupled equations involve the populations and the "linear coherences" ( $\left\langle\sigma_{13}\right\rangle$ and $\left\langle\sigma_{24}\right\rangle$ terms) which relate to the $z$ component of the atomic dipole (see eqn. 3).

$$
\begin{aligned}
\frac{d}{d t}\left\langle\sigma_{13}\right\rangle & =(-\gamma-\imath \Delta)\left\langle\sigma_{13}\right\rangle-\imath \Omega\left(\left\langle\sigma_{33}\right\rangle-\left\langle\sigma_{11}\right\rangle\right) \\
\frac{d}{d t}\left\langle\sigma_{31}\right\rangle & =(-\gamma+\imath \Delta)\left\langle\sigma_{31}\right\rangle+\imath \Omega\left(\left\langle\sigma_{33}\right\rangle-\left\langle\sigma_{11}\right\rangle\right) \\
\frac{d}{d t}\left\langle\sigma_{24}\right\rangle & =(-\gamma-\imath \Delta)\left\langle\sigma_{24}\right\rangle-\imath \Omega\left(\left\langle\sigma_{22}\right\rangle-\left\langle\sigma_{44}\right\rangle\right) \\
\frac{d}{d t}\left\langle\sigma_{42}\right\rangle & =(-\gamma+\imath \Delta)\left\langle\sigma_{42}\right\rangle+\imath \Omega\left(\left\langle\sigma_{22}\right\rangle-\left\langle\sigma_{44}\right\rangle\right) \\
\frac{d}{d t}\left\langle\sigma_{11}\right\rangle & =-\imath \Omega\left(\left\langle\sigma_{31}\right\rangle-\left\langle\sigma_{13}\right\rangle\right)+\gamma\left(\left\langle\sigma_{33}\right\rangle+\left\langle\sigma_{44}\right\rangle\right)
\end{aligned}
$$




$$
\begin{aligned}
& \frac{d}{d t}\left\langle\sigma_{22}\right\rangle=-\imath \Omega\left(\left\langle\sigma_{24}\right\rangle-\left\langle\sigma_{42}\right\rangle\right)+\gamma\left(\left\langle\sigma_{33}\right\rangle+\left\langle\sigma_{44}\right\rangle\right) \\
& \frac{d}{d t}\left\langle\sigma_{33}\right\rangle=-\imath \Omega\left(\left\langle\sigma_{13}\right\rangle-\left\langle\sigma_{31}\right\rangle\right)-2 \gamma\left\langle\sigma_{33}\right\rangle \\
& \frac{d}{d t}\left\langle\sigma_{44}\right\rangle=-\imath \Omega\left(\left\langle\sigma_{42}\right\rangle-\left\langle\sigma_{24}\right\rangle\right)-2 \gamma\left\langle\sigma_{44}\right\rangle .
\end{aligned}
$$

Since normalization requires that

$$
\sum_{i=1}^{4}\left\langle\sigma_{i i}\right\rangle=1,
$$

the number of independent equations may be reduced to seven. Setting the time derivatives to zero for the steady-state yields"

$$
\begin{aligned}
\left\langle\sigma_{13}\right\rangle_{s s} & =(\bar{\Delta}+\imath \bar{\gamma})\left\langle\sigma_{33}\right\rangle_{s s} \\
\left\langle\sigma_{31}\right\rangle_{s s} & =\left\langle\sigma_{13}\right\rangle_{s s}^{*} \\
\left\langle\sigma_{24}\right\rangle_{s s} & =(-\bar{\Delta}-\imath \bar{\gamma})\left\langle\sigma_{44}\right\rangle_{s s} \\
\left\langle\sigma_{42}\right\rangle_{s s} & =\left\langle\sigma_{24}\right\rangle_{s s}^{*} \\
\left\langle\sigma_{11}\right\rangle_{s s} & =\frac{1}{2}-\left\langle\sigma_{33}\right\rangle_{s s} \\
\left\langle\sigma_{22}\right\rangle_{s s} & =\left\langle\sigma_{11}\right\rangle_{s s} \\
\left\langle\sigma_{33}\right\rangle_{s s} & =\frac{1}{2}\left(\bar{\Delta}^{2}+\bar{\gamma}^{2}+2\right)^{-1} \\
\left\langle\sigma_{44}\right\rangle_{s s} & =\left\langle\sigma_{33}\right\rangle_{s s}
\end{aligned}
$$

where $\bar{\Delta}=\Delta / \Omega$ and $\bar{\gamma}=\gamma / \Omega$. As expected from the symmetry of the situation, the populations of the two ground states are equal as are the populations of the two excited states.

A graph of the populations versus laser light strength is shown in fig. 2(a). For strong fields the atom is saturated with all the populations equal to one quarter. For weak fields, the excited states are close to zero with the ground states close to one half. The linear coherences are shown in fig. 2 (b). Since the coherences are purely imaginary we plot the imaginary part of $\left\langle\sigma_{13}\right\rangle$ and $\left\langle\sigma_{24}\right\rangle$. We see that as the field strength increases the coherences grow and reach a peak for $\Omega / \gamma \approx 2 / 3$. There is a gradual loss of coherence as the field is further increased due to the saturation of the atom. At low field strengths the atom spends most of its time in the ground state and is very rarely pumped to the excited states. It behaves like a coherently driven linear oscillator and scatters coherently. In the strong field case, the atom undergoes Rabi oscillations and is frequently pumped to the excited states from which it spontaneously decays. These spontaneous emission events destroy the coherences.

The second set of equations consist of the "cross coherences" $\left(\left\langle\sigma_{14}\right\rangle\right.$ and $\left.\left\langle\sigma_{23}\right\rangle\right)$ and the coherences between the ground $\left\langle\sigma_{12}\right\rangle$ and between the excited states $\left\langle\sigma_{34}\right\rangle$.

$$
\begin{aligned}
\frac{d}{d t}\left\langle\sigma_{14}\right\rangle & =(-\gamma-\imath \Delta)\left\langle\sigma_{14}\right\rangle-\imath \Omega\left(\left\langle\sigma_{12}\right\rangle+\left\langle\sigma_{34}\right\rangle\right) \\
\frac{d}{d t}\left\langle\sigma_{23}\right\rangle & =(-\gamma-\imath \Delta)\left\langle\sigma_{23}\right\rangle+\imath \Omega\left(\left\langle\sigma_{21}\right\rangle+\left\langle\sigma_{43}\right\rangle\right) \\
\frac{d}{d t}\left\langle\sigma_{12}\right\rangle & =-\imath \Omega\left(\left\langle\sigma_{14}\right\rangle+\left\langle\sigma_{32}\right\rangle\right)-\gamma\left\langle\sigma_{34}\right\rangle \\
\frac{d}{d t}\left\langle\sigma_{34}\right\rangle & =-\imath \Omega\left(\left\langle\sigma_{32}\right\rangle+\left\langle\sigma_{14}\right\rangle\right)-2 \gamma\left\langle\sigma_{34}\right\rangle
\end{aligned}
$$

The other four equations for $\left\langle\sigma_{41}\right\rangle,\left\langle\sigma_{32}\right\rangle,\left\langle\sigma_{21}\right\rangle$ and $\left\langle\sigma_{43}\right\rangle$ are the Hermitian conjugates of these equations. Solving this set of equations in the steady-state we find that only the trivial solution remains so that all these coherences ultimately vanish. Any initial coherences involving these terms are damped in the transient regime. Since the cross coherences, $\left\langle\sigma_{14}\right\rangle$ and $\left\langle\sigma_{23}\right\rangle$, determine the $x$ and $y$ components of the atomic dipole (see eqn. 1 $1-3$ ), there is no interference from light polarized in the $x y$ plane.

\footnotetext{
${ }^{1}$ These solutions are compatible to those of ref [7] since only one atom has been included at this time.
} 


\section{THE INTERFERENCE PATTERN}

We have calculated in the previous section the steady-state solutions for the atomic coherences and populations for the case of a single four-level atom interacting with a classical laser light field linearly polarized along the $z$ axis. In this section we wish to use these to calculate the far-field interference pattern from two such atoms in the case when their separation is large enough that they may be treated independently.

Let us consider a screen placed in the far-field (large $y$ ) and oriented in the $x z$ plane. In the far-field, and in the paraxial approximation, the electric field at a point on the screen at time $t$ due to an oscillating dipole is proportional to the projection onto the plane of the screen of the acceleration of the dipole moment at the retarded time $t-\tau$, where $\tau$ is the light travel time from the dipole to the observation point. We shall label a point on the screen by $\left(\tau_{1}, \tau_{2}\right)$ where these are the light travel times from each of the atoms to the point on the screen. The intensity of the light at this point is

$$
I\left(\tau_{1}, \tau_{2}\right) \propto\left\langle E_{x}^{\uparrow} E_{x}^{\downarrow}+E_{z}^{\uparrow} E_{z}^{\downarrow}\right\rangle
$$

where

$$
E_{k}^{\uparrow}\left(t ; \tau_{1}, \tau_{2}\right) \propto \mathrm{e}^{-i \omega\left(t-\tau_{1}\right)} \mu_{k}^{\uparrow}+\mathrm{e}^{-i \omega\left(t-\tau_{2}\right)} \mathcal{U}_{k}^{\uparrow}
$$

for $k \in\{x, z\}, \mu$ and $\mathcal{U}$ are the atomic dipoles of the first and second atoms respectively and $\omega$ is the angular frequency of the laser light. If we use the notation $\sigma_{i j}$ for the atomic operators of the first atom and $\Sigma_{i j}$ for those the second, we note that the cross terms like $\left\langle\mu_{x}^{\uparrow} \mathcal{U}_{x}^{\downarrow}\right\rangle$ are zero in the steady-state since

$$
\begin{aligned}
\left\langle\mu_{x}^{\uparrow} \mathcal{U}_{x}^{\downarrow}\right\rangle_{s s} & \propto\left\langle\left(\sigma_{41}+\sigma_{32}\right)\left(\Sigma_{14}+\Sigma_{23}\right)\right\rangle_{s s} \\
& =\left(\left\langle\sigma_{41}\right\rangle_{s s}+\left\langle\sigma_{32}\right\rangle_{s s}\right)\left(\left\langle\Sigma_{14}\right\rangle_{s s}+\left\langle\Sigma_{23}\right\rangle_{s s}\right) \\
& =0 .
\end{aligned}
$$

We have factorized the products above since we have assumed that the atoms are independent. The intensity of the interference pattern when all the light is detected is given by

$$
\begin{aligned}
I_{\text {unpol }}\left(\tau_{1}, \tau_{2}\right) \propto & \left\langle\mu_{x}^{\uparrow} \mu_{x}^{\downarrow}+\mathcal{U}_{x}^{\uparrow} \mathcal{U}_{x}^{\downarrow}+\mu_{z}^{\uparrow} \mu_{z}^{\downarrow}+\mathcal{U}_{z}^{\uparrow} \mathcal{U}_{z}^{\downarrow}\right\rangle \\
& +\left\langle\mu_{z}^{\uparrow} \mathcal{U}_{z}^{\downarrow}\right\rangle \exp \left(\imath\left[\omega\left(\tau_{1}-\tau_{2}\right)-\phi_{o}\right]\right) \\
& +\left\langle\mathcal{U}_{z}^{\uparrow} \mu_{z}^{\downarrow}\right\rangle \exp \left(-\imath\left[\omega\left(\tau_{1}-\tau_{2}\right)-\phi_{o}\right]\right)
\end{aligned}
$$

where the additional phase term $\phi_{o}$ has been included to allow for the phase of the incident light to differ at the positions of the two ions. If this is normalized by twice the intensity due to single atom fluorescence, we obtain

$$
I_{\mathrm{N}, \text { unpol }}\left(\tau_{1}, \tau_{2}\right)=1+V_{\text {unpol }} \cos \left[\omega\left(\tau_{1}-\tau_{2}\right)-\phi_{o}\right]
$$

where

$$
V_{\text {unpol }}=\frac{1}{2}\left(\frac{\bar{\Delta}^{2}+\bar{\gamma}^{2}}{\bar{\Delta}^{2}+\bar{\gamma}^{2}+2}\right)
$$

We note that this interference pattern is the sum of an incoherent term and a coherent term and so the visibility is always less than one half. If we introduce a polarizer at angle $\eta$ to the $z$ axis in front of the screen, it is possible to separate out the two components. The expression for the intensity is then

$$
\begin{aligned}
I_{\mathrm{pol}}\left(\tau_{1}, \tau_{2} ; \eta\right) \propto & \left\langle\mu_{x}^{\uparrow} \mu_{x}^{\downarrow}+\mathcal{U}_{x}^{\uparrow} \mathcal{U}_{x}^{\downarrow}\right\rangle \sin ^{2} \eta+\left\langle\mu_{z}^{\uparrow} \mu_{z}^{\downarrow}+\mathcal{U}_{z}^{\uparrow} \mathcal{U}_{z}^{\downarrow}\right\rangle \cos ^{2} \eta \\
& +\left\langle\mu_{z}^{\uparrow} \mathcal{U}_{z}^{\downarrow}\right\rangle \exp \left(\imath\left[\omega\left(\tau_{1}-\tau_{2}\right)-\phi_{o}\right]\right) \cos ^{2} \eta \\
& +\left\langle\mathcal{U}_{z}^{\uparrow} \mu_{z}^{\downarrow}\right\rangle \exp \left(-\imath\left[\omega\left(\tau_{1}-\tau_{2}\right)-\phi_{o}\right]\right) \cos ^{2} \eta
\end{aligned}
$$

Normalizing this as before yields

$$
I_{\mathrm{N}, \mathrm{pol}}\left(\tau_{1}, \tau_{2} ; \eta\right)=1+V_{\mathrm{pol}}(\eta) \cos \left[\omega\left(\tau_{1}-\tau_{2}\right)-\phi_{o}\right]
$$

where the visibility is now given by 


$$
V_{\mathrm{pol}}(\eta)=\left(\frac{\bar{\Delta}^{2}+\bar{\gamma}^{2}}{\bar{\Delta}^{2}+\bar{\gamma}^{2}+2}\right) \cos ^{2} \eta .
$$

Thus if we detect the $x$ polarized light by setting $\eta=90^{\circ}$, we see that the visibility is zero for any field strength and detuning. On the other hand if the polarizer is set along the $z$ axis $(\eta=0)$ then the visibility is non-zero. This visibility is plotted against field strength in fig. 3 for various detunings with the polarizer aligned with the $z$ axis $(\eta=0)$. The maximum theoretical visibility of one occurs for all detunings in the limit of zero field strength. As the field strength is increased the visibility falls towards zero, with the detuning determining the rate of this drop.

\section{COMPARISON WITH EXPERIMENTAL PARAMETERS}

In the experiment of Eichmann et al. [6] the incident laser is linearly polarized at an angle of $62^{\circ}$ degrees to the $z$ axis in the $y z$ plane. This gives a $y$ component to the atomic dipole which is not considered in the above calculation, but this does not affect the field on a screen in the $x z$ plane. The inclination also introduces a non-zero phase difference $\phi_{o}$ between the two atoms which has be included in our calculations. For more general orientations of the incident field, we resort to numerical simulations as described later 2 . Another difference between our analysis and the experiment is that instead of rotating a polarizer in the output field, a fixed Brewster plate is used and the polarization of the incident light is changed. Our analysis is not valid for incident light polarized along the $x$ direction, however our use of a rotating polarizer gives some insight into the polarization-sensitive measurement.

Taking into account the geometry of the experiment, we expect that the measured interference pattern should be

$$
I_{\mathrm{N}, \text { unpol }}=1+\frac{1}{2}\left(\frac{\bar{\Delta}^{2}+\bar{\gamma}^{2}}{\bar{\Delta}^{2}+\bar{\gamma}^{2}+2}\right) \cos \left[\left(\frac{2 \pi d}{\lambda}\right) \sin \left(\phi-28^{\circ}\right)-\phi_{o}\right]
$$

when all the light is collected and

$$
I_{\mathrm{N}, \mathrm{pol}}=1+\cos ^{2} \eta\left(\frac{\bar{\Delta}^{2}+\bar{\gamma}^{2}}{\bar{\Delta}^{2}+\bar{\gamma}^{2}+2}\right) \cos \left[\left(\frac{2 \pi d}{\lambda}\right) \sin \left(\phi-28^{\circ}\right)-\phi_{o}\right]
$$

when a polarization-sensitive measurement is made. Where $d$ is the distance between the atoms, $\phi$ is angle relative to the incident light and $\lambda$ is the wavelength of this light.

The visibility measured in the experiment when all the light was collected had a value of 0.2 . The maximum visibility from eqn. 36 is one half. This less than "ideal" measured visibility is not surprising since many detrimental factors have not been included in the analytical treatment. Factors such as stray light entering the detector and quantum jumps to other levels in the Mercury ions so that we may observe only the fluorescence from one ion. 8] The motion of the ions in the trap also smear out the interference pattern, this will be discuss in detail in a later section.

\section{NUMERICAL SIMULATION OF THE SYSTEM}

In this section, we describe the use of a Monte Carlo wave function method for numerical simulation of the system. This approach allows greater flexibility in specifying the geometry of the incident light and also allows us to include the photodetection process as part of the simulation. By doing this, we no longer simply relate the far field intensity of the fluorescent light to the moments of the atomic variables, but explicitly consider how atomic quantum jumps introduce photons into electromagnetic field modes propagating in various directions. The advantage of this approach is that it allows the atoms to be separated by arbitrary distances and takes into account the dipole-dipole correlations between the atoms. This simulation approach is very closely related to the actual experimental situation as it gives a time-resolved classical record of photocounts at different angles which gradually build up into an interference pattern.

For simplicity, we assume idealized unit efficiency photodetectors in the far field covering the entire $4 \pi$ steradians surrounding the two atoms. Each detector is assumed to be polarization-sensitive and resolves detected photons into

\footnotetext{
${ }^{2} \mathrm{~A}$ numerical simulation will be introduced later which can simulate the geometry of the incident light in a more flexible manner.
} 
two orthogonal linear polarizations. Working in spherical polar coordinates with polar angle $\theta$ and azimuthal angle $\phi$ (see fig. (1), we use the unit vectors $\hat{\epsilon}_{\theta}$ and $\hat{\epsilon}_{\phi}$ as a basis for the polarization of the emitted photon. These unit vectors are related to Cartesian coordinates by

$$
\begin{aligned}
& \hat{\epsilon}_{\theta}=\cos \theta \cos \phi \hat{\mathbf{x}}+\cos \theta \sin \phi \hat{\mathbf{y}}-\sin \theta \hat{\mathbf{z}} \\
& \hat{\epsilon}_{\phi}=-\sin \phi \hat{\mathbf{x}}+\cos \phi \hat{\mathbf{y}}
\end{aligned}
$$

where $\hat{\mathbf{x}}, \hat{\mathbf{y}}$ and $\hat{\mathbf{z}}$ are the Cartesian unit vectors. The system Hamiltonian for the two atoms is just the sum of the individual Hamiltonians for each atom

$$
\begin{aligned}
\mathrm{H}_{\text {sys }}= & \frac{1}{2} \hbar \Delta\left(\sigma_{44}+\sigma_{33}-\sigma_{22}-\sigma_{11}+\Sigma_{44}+\Sigma_{33}-\Sigma_{22}-\Sigma_{11}\right) \\
& +\hbar \Omega_{z}\left(\sigma_{24}+\sigma_{42}-\sigma_{13}-\sigma_{31}+\Sigma_{24}+\Sigma_{42}-\Sigma_{13}-\Sigma_{31}\right) \\
& +\hbar \Omega_{+}\left(\sigma_{41}+\sigma_{23}+\Sigma_{41}+\Sigma_{23}\right)+\hbar \Omega_{-}\left(\sigma_{14}+\sigma_{32}+\Sigma_{14}+\Sigma_{32}\right),
\end{aligned}
$$

where we have set $\Omega_{ \pm}=\Omega_{x} \pm \imath \Omega_{y}$, and where $\Omega_{x}, \Omega_{y}$ and $\Omega_{z}$ are the Rabi frequencies corresponding to the $x, y$, and $z$ components of the electric field

$$
\Omega_{k}=\frac{\mu \vec{E}_{k}}{2 \hbar}, \quad k=x, y, z,
$$

where $\mu$ is the dipole matrix element and each atom is considered to feel the same electric field $\vec{E}$. We are assuming here that the incident light is propagating at right angles to the line joining the two atoms. The relaxation superoperator is in Lindblad form

$$
\mathcal{L}_{\text {relax }}\left(\rho_{s}\right)=-\frac{1}{2} \sum_{m}\left(C_{m}^{\dagger} C_{m} \rho_{s}+\rho_{s} C_{m}^{\dagger} C_{m}\right)+\sum_{m} C_{m} \rho_{s} C_{m}^{\dagger}
$$

where the collapse operators $C_{m}$ correspond to the couplings between system and the baths. When the atoms are far apart, they may be regarded as coupling to independent baths and so collapses associated with one atom may be distinguished from those associated with the other. If we explicitly include the photodetection scheme, the appropriate collapse operators are parameterized by the direction and polarization of the outgoing photon. For spontaneous emission from a single two-level atom, the appropriate collapse operators (including recoil) are [9]

$$
C_{\Omega, \hat{\epsilon}}=\left(\frac{3 \gamma}{8 \pi}\right)^{1 / 2} \exp (-\imath \overrightarrow{\mathbf{k}} \cdot \overrightarrow{\mathbf{R}})\left(\hat{\epsilon}^{*} \cdot \overrightarrow{\mathbf{S}}^{\downarrow}\right)
$$

where $\overrightarrow{\mathbf{R}}$ is the atomic position operator, $\overrightarrow{\mathbf{k}}$ is the wave number of the outgoing photon traveling in direction $\Omega, \overrightarrow{\mathbf{S}}^{\downarrow}$ is an operator proportional to the atomic lowering operator $\left(\overrightarrow{\mathbf{S}}^{\downarrow} \propto \sigma^{-}\right)$and $\hat{\epsilon}$ labels the outgoing polarization. For our situation with two fixed atoms, $\overrightarrow{\mathbf{R}}$ may be replaced by the locations of the two atoms $\overrightarrow{\mathbf{r}}_{1}$ and $\overrightarrow{\mathbf{r}}_{2}$ (see fig. 目) and both atoms couple to each mode so that

$$
C_{\Omega, \epsilon}=\mathcal{N}\left\{\exp \left(-\imath \overrightarrow{\mathbf{k}} \cdot \overrightarrow{\mathbf{r}}_{1}\right)\left(\hat{\epsilon}^{*} \cdot \overrightarrow{\mathbf{S}}_{1}^{\downarrow}\right)+\exp \left(-\imath \overrightarrow{\mathbf{k}} \cdot \overrightarrow{\mathbf{r}}_{2}\right)\left(\hat{\epsilon}^{*} \cdot \overrightarrow{\mathbf{S}}_{2}^{\downarrow}\right)\right\}
$$

where $\mathcal{N}$ is a normalization factor. $\overrightarrow{\mathbf{S}}_{1}^{\downarrow}$ and $\overrightarrow{\mathbf{S}}_{2}^{\downarrow}$ are proportional to the atomic dipole operators for the first and second atoms respectively. The Cartesian components of these operators are

$$
\begin{aligned}
& \overrightarrow{\mathbf{S}}_{1}^{\downarrow} \propto \sigma_{x} \hat{\mathbf{x}}+\sigma_{y} \hat{\mathbf{y}}+\sigma_{z} \hat{\mathbf{z}} \\
& \overrightarrow{\mathbf{S}}_{2}^{\downarrow} \propto \Sigma_{x} \hat{\mathbf{x}}+\Sigma_{y} \hat{\mathbf{y}}+\Sigma_{z} \hat{\mathbf{z}}
\end{aligned}
$$

where the $\sigma_{i}$ 's are given by

$$
\begin{aligned}
\sigma_{x} & =\sigma_{14}+\sigma_{23} \\
\sigma_{y} & =-\imath\left(\sigma_{14}-\sigma_{23}\right) \\
\sigma_{z} & =\sigma_{24}-\sigma_{13},
\end{aligned}
$$

and the $\Sigma_{i}$ 's related to the $\Sigma_{i j}$ 's in a similar way. We see explicitly from these expressions that when a quantum jump (i.e., a photodetection) occurs, this may come from either atom, the coefficient being dependent on the polarization and the appropriate phase factor. 
Between the times of the quantum jumps, the atomic wave function is evolved using the non-Hermitian effective Hamiltonian given by 3

$$
\mathbf{H}_{e f f}=\mathbf{H}_{s y s}-\frac{\imath \hbar}{2} \sum_{m} C_{m}^{\dagger} C_{m}
$$

On the right-hand side, the summation is responsible for the decrease in the norm of the Monte-Carlo wave function. Its expectation value is proportional to the intensity of the fluorescent light and the probability that a quantum jump occurs. For our photodetection model, this eventually evaluates to

$$
\begin{aligned}
\int \sum_{\hat{\epsilon} \perp \overrightarrow{\mathrm{k}}} C_{\Omega, \hat{\epsilon}}^{\dagger} C_{\Omega, \hat{\epsilon}} \mathrm{d} \Omega= & \frac{8 \pi}{3} \mathcal{N}^{2}\left\{\left(\sigma_{x}^{\dagger} \sigma_{x}+\sigma_{y}^{\dagger} \sigma_{y}+\sigma_{z}^{\dagger} \sigma_{z}+\Sigma_{x}^{\dagger} \Sigma_{x}+\Sigma_{y}^{\dagger} \Sigma_{y}+\Sigma_{z}^{\dagger} \Sigma_{z}\right)\right. \\
& +\mathcal{A}(\alpha)\left(\sigma_{x}^{\dagger} \Sigma_{x}+\sigma_{y}^{\dagger} \Sigma_{y}+\Sigma_{x}^{\dagger} \sigma_{x}+\Sigma_{y}^{\dagger} \sigma_{y}\right) \\
& \left.+\mathcal{B}(\alpha)\left(\sigma_{z}^{\dagger} \Sigma_{z}+\Sigma_{z}^{\dagger} \sigma_{z}\right)\right\}
\end{aligned}
$$

where

$$
\begin{aligned}
\mathcal{A}(\alpha) & =\frac{3}{8}\left(\frac{4}{\alpha} \sin \alpha-\mathcal{T}(\alpha)\right) \\
\mathcal{B}(\alpha) & =\frac{3}{4} \mathcal{T}(\alpha)
\end{aligned}
$$

where $\alpha=|\overrightarrow{\mathbf{k}}| \cdot\left|\overrightarrow{\mathbf{r}}_{2}-\overrightarrow{\mathbf{r}}_{1}\right|$ is the $2 \pi$ times the separation between the two atoms in optical wavelengths. The function $\mathcal{T}$ is given by

$$
\mathcal{T}(\alpha)=\frac{4}{\alpha^{2}}\left(\frac{1}{\alpha} \sin \alpha-\cos \alpha\right) .
$$

The graph of the functions $\mathcal{A}$ and $\mathcal{B}$ are shown in fig. 5 . Note that we have used the scaled parameter $\bar{\alpha}=\alpha / 2 \pi$ corresponding to units of wavelengths. At $\bar{\alpha}$ equal to zero where the two atoms coincide, both functions are equal to one. As the separation between the atoms is increased both display damped oscillatory behavior decaying to zero in the limit of large separations. $\mathcal{B}$ is damped more rapidly and is almost zero for separations of more than three wavelengths, whereas $\mathcal{A}$ has larger amplitude oscillations which can still be clearly seen even after six wavelengths. Notice that $\mathcal{B}$ corresponds to the size of the correlations between the $z$ components of the atomic dipoles whereas $\mathcal{A}$ determines the size of the correlations between the $x$ and $y$ components. In the limit of large separation between the two atoms, only the first term of eqn. 51 remains and we have

$$
\begin{aligned}
{\left[\int \sum_{\hat{\epsilon} \perp \overrightarrow{\mathrm{k}}} C_{\Omega, \hat{\epsilon}}^{\dagger} C_{\Omega, \hat{\epsilon}} \mathrm{d} \Omega\right]_{\alpha \rightarrow \infty} } & =\mathcal{N}^{2} \frac{8 \pi}{3}\left(\sigma_{x}^{\dagger} \sigma_{x}+\sigma_{y}^{\dagger} \sigma_{y}+\sigma_{z}^{\dagger} \sigma_{z}+\Sigma_{x}^{\dagger} \Sigma_{x}+\Sigma_{y}^{\dagger} \Sigma_{y}+\Sigma_{z}^{\dagger} \Sigma_{z}\right) \\
& \equiv \mathcal{N}^{2} 8 \pi\left(\sigma_{44}+\sigma_{33}+\Sigma_{44}+\Sigma_{33}\right) .
\end{aligned}
$$

This may be compared with the form expected for two atoms coupling to independent baths for which

$$
\sum_{m} C_{m}^{\dagger} C_{m}=2 \gamma\left(\sigma_{44}+\sigma_{33}+\Sigma_{44}+\Sigma_{33}\right)
$$

We see that in the limit of large separation, the atoms behave independently, and that the normalization condition is $\mathcal{N}=(\gamma / 4 \pi)^{1 / 2}$. In the opposite limit of the two atoms coinciding

\footnotetext{
${ }^{3}$ see $\mathrm{M} \phi$ lmer et al. $[9$

${ }^{4}$ The function $\mathcal{T}(\alpha)$ has similar structural form to the dipole-dipole term used by T.G. Rudolph et al. [10] and J. Guo et al. [11] to modify the decay rate of the atoms.
} 


$$
\begin{aligned}
{\left[\int \sum_{\hat{\epsilon} \perp \overrightarrow{\mathrm{k}}} C_{\Omega, \hat{\epsilon}}^{\dagger} C_{\Omega, \hat{\epsilon}} \mathrm{d} \Omega\right]_{\alpha \rightarrow 0}=} & \mathcal{N}^{2} \frac{8 \pi}{3}\left(\sigma_{x}^{\dagger} \sigma_{x}+\sigma_{y}^{\dagger} \sigma_{y}+\sigma_{z}^{\dagger} \sigma_{z}+\Sigma_{x}^{\dagger} \Sigma_{x}+\Sigma_{y}^{\dagger} \Sigma_{y}+\Sigma_{z}^{\dagger} \Sigma_{z}\right. \\
& \left.+\sigma_{x}^{\dagger} \Sigma_{x}+\Sigma_{x}^{\dagger} \sigma_{x}+\sigma_{y}^{\dagger} \Sigma_{y}+\Sigma_{y}^{\dagger} \sigma_{y}+\sigma_{z}^{\dagger} \Sigma_{z}+\Sigma_{z}^{\dagger} \sigma_{z}\right) \\
\equiv & \mathcal{N}^{2} \frac{8 \pi}{3}\left(\left|\sigma_{x}+\Sigma_{x}\right|^{2}+\left|\sigma_{y}+\Sigma_{y}\right|^{2}+\left|\sigma_{z}+\Sigma_{z}\right|^{2}\right)
\end{aligned}
$$

This gives four times the decay rate of a single atom instead of two times for the case of large separation since the interactions between the atoms grow as the separation decreases until at zero separation the size of these dipole-dipole terms become of the same order as the population terms. This gives rise to superfluorescence where the two atoms behave as one. This is clearly illustrated by the factorization of the above expression into the coherent sum of the two atoms.

In order to carry out the Monte Carlo simulation, a wave function is evolved using the Schrödinger equation with the non-Hermitian effective Hamiltonian until the square of the norm of the wave function reaches a threshold drawn from a uniform distribution lying between zero and one. At this time, it is necessary to select one of the possible quantum jumps. The probability density of detecting a fluorescence photon traveling in direction $(\theta, \phi)$ with polarization $\epsilon_{i}$ is given by

$$
\mathcal{P}_{\epsilon_{i}}(\theta, \phi)=\frac{\left\langle C_{\Omega, \epsilon_{i}}^{\dagger} C_{\Omega, \epsilon_{i}}\right\rangle}{\left\langle\int \sum_{\epsilon \perp \overrightarrow{\mathrm{k}}} C_{\Omega, \epsilon}^{\dagger} C_{\Omega, \epsilon} \mathrm{d} \Omega\right\rangle} \quad i=\theta \text { or } \phi .
$$

Conventionally, a Monte Carlo simulation requires many runs to be carried out to obtain the desired statistics of the system from an ensemble of quantum trajectories. For the problem being considered, the result of one quantum trajectory gives the photo-emission record for the two atom system, and we can let this single trajectory run as long as it is necessary to obtain the required number of photons.

\section{NUMERICAL RESULTS}

The raw output of these simulations is a list of the emission times, directions and polarizations of each emitted photon. The direction is represented by the two angles $\theta$ and $\phi$, with the polarization aligned either with the unit vector $\epsilon_{\theta}$ or $\epsilon_{\phi}$.

The wavelength of the incident laser light used in all of the following numerical simulations was 194nm. In the first simulation we have chosen parameters which gave maximum visibility of the interference pattern. The laser field was weak, the ratio of Rabi frequency to decay rate being 0.2 . The atomic separation was set at $0.5 \mu m$ so that five intensity peaks would be observed as $\theta$ is varied through $180^{\circ}$. This gives a sufficient number of points in order to clearly distinguish each peak with the number of photodetections simulated. The incident light is propagating along the $y$ axis.

The intensity pattern as seen on a sphere around the ions is shown in fig. 6 for incident light polarized in the $z$ direction. Since the system is symmetric about the $z$ axis, fig. 6 (a) shows an interference pattern as $\theta$ (but not $\phi$ ) is varied. Fig. 6(b) is another simulation in which the incident light is linearly polarized along the $x$ axis. Even though the system is no longer symmetric about the $z$ axis, there is little dependence of the interference on the angle $\phi$. We shall henceforth only plot the interference patterns as functions of $\theta$, and integrate over the angle $\phi$. This integration over $\phi$ modulates the intensity distribution with a sinusoidal envelope since the solid angle integrated over at the equator $\left(\theta=90^{\circ}\right)$ is greater that at the poles $\left(\theta=0^{\circ}\right.$ or $\left.180^{\circ}\right)$.

For the case of incident light polarized in the $z$ direction, fig. 7(a) shows the intensity distribution for $\epsilon_{\theta}$ polarized light as a function of angle $\theta$ from a simulation consisting of one hundred thousand quantum jumps. Out of these, about half were detected with $\epsilon_{\theta}$ polarization. The histogram ${ }^{\circ}$ is computed using a bin width of $1^{\circ}$. An interference pattern is clearly visible. From eqn. 35, we expect a visibility of $25 / 27$ or about 0.9 but the visibility measured from the simulation is approximately 0.7 . However eqn. 35 is only valid in the paraxial approximation and only considers photons polarized in the $z$ direction. When this approximation breaks down (i.e., when $\theta$ is no longer close to $90^{\circ}$ ), the $\epsilon_{\theta}$ polarization will include contributions from the components of the atomic dipole other than $\sigma_{z}$. These will

\footnotetext{
${ }^{5}$ We have plotted the intensity distributions as histograms without the internal lines for clarity.
} 
reduce the total visibility as we do not expect interference from the $x$ and $y$ components of the atomic dipole which are not coherently driven. If we now look at the orthogonal polarization (the $\epsilon_{\phi}$ polarization) as shown in see fig. If(b), there is no interference pattern. The $\epsilon_{\phi}$ polarization is always perpendicular to the $z$ axis for any angle $\theta$ so only the $x$ and $y$ components of the atomic dipoles contribute.

We now consider the case where the incident laser light is polarized along the $x$ axis instead of the $z$ axis. The histogram for the intensity of $\epsilon_{\theta}$ polarized light versus $\theta$ is shown in fig. 8(a). There is no obvious interference pattern now since the $\theta$ polarization consists mainly of the $z$ component of the atomic dipole for angles around $\theta=90^{\circ}$. Small peaks can be seen at about 40 and 140 degrees, which are due to the $x$ component of the atomic dipole which contribute in larger amounts as the $\theta$ deviates significantly from 90 degrees. Fig. 8(b) displays interference as expected since the $\phi$ polarization only consists of the $x$ and $y$ components of the atomic dipole. Since the incident light is linearly polarized along the $x$ axis, the $y$ component of the scattered light is not coherent and the visibility should be less than seen in 7 (a). From the central peaks the visibility is about one third, approximately half that for the case of light polarized in the $z$ direction.

\section{EFFECTS OF MOTION}

We have so far ignored the effects of the motion of the ions as this has been secondary to our goal of demonstrating how the visibility of the interference pattern varies with polarization. However it is a significant effect in the experiment performed by Eichmann et al. We shall model the motion of the ions as harmonic oscillators for each of the three main modes, one involving stretching and two involving tilting [6, 12]. The stretching mode involves motion of the atoms along the $z$ axis whereas the two tilting modes involve motion in the $x y$ plane. Thus the stretching mode alters the distance between the ions and the period of the interference pattern while the tilting modes change the angle $\theta$ of the photon emission displacing the interference pattern. Both of these effects smear the interference pattern, reducing the visibility.

The temperature of the ions in the trap were around the milli-Kelvin region and the frequency of oscillation of the modes were of order of a MHz. By comparing the thermal kinetic energy of an ion $k_{\mathrm{B}} T / 2$ with the separation of the trap energy levels, $\hbar \omega$, we find that $k_{\mathrm{B}} T /(2 \hbar \omega) \approx 10^{2}$ and so the motion may be treated classically. The ions experience micromotion within the trap and the recoil momentum of the photon emissions may be neglected.

In the actual experiment, the values used for the separation between the ions was around $5 \mu m$. These values are about ten times larger than the values used in the simulations. Thus we expect to see over fifty peaks over a range of one hundred and eighty degrees. We carried out a simulation with an ion separation of $3.35 \mu m$, where one hundred thousand jumps was performed. In fig. 9(a), we show the results for incident light which is linearly polarized along the $z$ axis, with no motion of the ions. The peaks are poorly resolved and an excessive length of time would be required to obtain sharper resolution for such finely separated peaks. Note that in this graph, the detector is taken to be polarization insensitive and we count all the photons. Fig. 9(a) shows the correct period (nine peaks from 75 to 105 degrees) with a visibility of about 0.35 which is lower than the predicted value of 0.46 using eqn. 32 .

The geometry of the incident light in the simulation differs from the that of the experiment. The light is propagating along the $y$ axis in the simulation whereas the experiment has the incident light at an angle of $62^{\circ}$ relative to the $z$ axis. However, the experiment does not detect light over the entire $4 \pi$ steradians, only a small portion is actually observed. This observation region is a small disk perpendicular to the $y$ axis. By summing over the $\phi$ angle in the simulation and considering only $\theta$ angles close to the $y$ axis we have an approximation of the experimentally observed region of interest.

The micromotion is simulated in two ways. We keep track of the oscillatory motion of the stretch mode starting with some arbitrary initial value. Since we are interested in the long time (steady-state) regime the actual initial value we choose is unimportant. The previous numerical procedure is re-used but the atomic separation is altered for every photon emission according to the displacement of the stretch mode. This is valid because the characteristic time scale of the stretch mode $\tau_{\text {stretch }}\left(\sim 10^{-6} s\right)$ is far longer than the atomic half-life $\tau_{1 / 2}\left(\sim 10^{-8}\right)$. The steady-state regime is satisfied since the duration of a simulation, $\tau_{\text {sim }}$, producing one hundred thousand photodetections is approximately $10^{-3} s$ which is much longer than the time scale of the stretch mode. The effects of the tilting motion is to shift the interference pattern. Thus during the simulation whenever we calculate a photodetection, the direction of the photon needs to be shifted according to the current displacement of the tilting modes. The $x$ and $y$ displacements are randomly generated from a harmonic oscillator probability distribution. In hindsight we could have generated $z$ displacements from a harmonic oscillator distribution as well, however in the simulations we have chosen to keep track of the stretch mode starting from some arbitrary value. Both methods are equivalent for the steady-state regime. The requirements on the time scales involved is

$$
\tau_{\text {sim }} \gg \tau_{\text {stretch }} \gg \tau_{1 / 2} .
$$


As expected, when classical motion is included in the simulation, the visibility of the interference is reduced, see fig. 9(b). We see an interference pattern with the peaks placed in the same positions as fig. 9(b) but with a reduced visibility of about 0.28 . The visibility for individual peaks degrades as one deviates from the central peak at ninety degrees. This maybe explained by the fact that we have modeled the motion along the $z$ axis as a stretching where the center-of-mass remains stationary, thus the central peak is in the same position whatever the period. As one deviates to the sides, a small change in period has a increasingly larger effect on the position of the peak. The parameters used in this simulation for the motion were: stretch frequency $\omega_{\text {stretch }} / 2 \pi=\sqrt{3} \mathrm{MHz}$, tilt frequencies $\omega_{\text {tilt }} / 2 \pi=\sqrt{5} / 2 \mathrm{MHz}$ (for both modes), radial confinement was $30 \mathrm{~nm}$ and the amplitude of the stretch mode was $150 \mathrm{~nm}$. Experimental results were shown in the paper by Eichmann et al. for this set of parameters. The visibility was about 0.2 which is less than the value obtained in the simulation. The greater visibility in the simulation is not unexpected since the effects of stray light and the fact that ${ }^{198} \mathrm{Hg}^{+}$ions are not ideal four-level atoms would further reduce the visibility.

\section{THE TRANSIENT REGIME}

So far we have investigated the steady-state regime which corresponds to the experimental situation. However the transient regime is also interesting because it is then possible to consider the which-path argument which explains the disappearance of an interference pattern as being due to information of which atom has undergone an emission being somehow recorded in the system. This knowledge can be obtained for some initial atomic states but not others.

In this section, we consider that the two atoms are initialized to some known state and then are exposed to the incident light. When the first fluorescence photon is detected, the experiment is stopped and the atoms are reinitialized to the original state. Over many trials, an interference pattern may develop in the far field, and it is this pattern which is of interest.

Let us first consider the case where the initial joint atomic state is $|\psi\rangle=|1,1\rangle$ and the incident light is polarized in the $z$ direction so that the $1 \leftrightarrow 3$ transition is driven. When one of the atoms has been excited to the state 3 , it may decay either to the state 1 or to the state 2 . If the decay is to state 1 , the final state of the atoms is again $|1,1\rangle$ and it is not possible to tell from which atom the light came from. We thus expect that the detection of light polarized in the $\epsilon_{\theta}$ direction which is sensitive to radiation from the $3 \rightarrow 1$ transition will exhibit interference. On the other hand, if the excited atom decays to state 2 , the final state of the atoms is either $|2,1\rangle$ or $|1,2\rangle$, leaving a record of the atom responsible for the emission. We thus expect light detected with polarization in the $\epsilon_{\phi}$ direction to not exhibit any interference at all.

The results of a simulation with this initial state are shown in fig. 10(a) for $\epsilon_{\theta}$ polarization and in fig. 10(b) for $\epsilon_{\phi}$ polarization. The atomic separation was $500 \mathrm{~nm}$ and the ratio of Rabi frequency to decay rate is 0.2 as before. As expected, a very distinct interference pattern can be seen in (a) consisting of five peaks. The visibility is about 0.88 , this is quite close to the expected theoretical value of about 0.9 . For the same set of parameters this transient simulation gives higher visibility than the corresponding steady-state simulation. Also as expected, no interference is seen in (b).

Let us now consider the initial joint atomic state $|\psi\rangle=(|1,1\rangle+|1,2\rangle+|2,1\rangle+|2,2\rangle) / 2$. In this case, it is not possible to distinguish the atom responsible for emitting the photon whether it is detected in the $\epsilon_{\theta}$ or the $\epsilon_{\phi}$ polarizations. We thus expect to see interference for both polarizations. The result of the simulation for the $\epsilon_{\phi}$ polarization is shown in fig. 11, confirming that this interference does occur in the transient regime. As discussed previously however, since the driving field is polarized in the $z$ direction, this coherence must approach zero in the steady-state and there can be no steady-state interference pattern, although interference does occur in the transient regime.

We have used extreme parameters so far, for the case of the $|1,1\rangle$ initial state we know for certain that each atom is initially in a particular ground state and for the other initial state $(|\psi\rangle=(|1,1\rangle+|1,2\rangle+|2,1\rangle+|2,2\rangle) / 2)$ we have no initial preference for which ground state each of the atoms are in. In fig. 12 results for the $\epsilon_{\phi}$ polarization are shown for an initial state $|\psi\rangle=(2|1,1\rangle+|1,2\rangle+|2,1\rangle+|2,2\rangle) / \sqrt{7}$. In this intermediate case, there is some visibility in the transient interference pattern, but this is only about a third, lower than that seen in the previous case.

In the transient regime we expect interference in the undriven polarization which does not appear in the steady-state regime for the majority of the possible initial states. The interference we see here is due to the presence of initial coherences. It is similar to the case of two two-level atoms prepared in the excited states, one would eventually decay emitting a photon in the process. The atoms are then re-prepared in the excited states and the process is repeated to build up an intensity pattern. It is not important in this regime whether there is coherent driving or not but only that the atoms are excited to higher levels thus giving them an opportunity to undergo spontaneous emission. 


\section{SUMMARY}

We have analyzed the experiment by Eichmann et al. and presented theoretical models for understanding the degree of interference observed for different incident and detected polarizations. For a simple geometry of the incident field, it is possible to solve the optical Bloch equations analytically for a single four-level atom in the steady-state, and to extend this result to the case of two atoms, assuming that they radiate independently. From this analysis, the fluorescent light is seen to consist of a coherent component which exhibits interference and an incoherent component which does not. These components may be separated out by polarizers before the light is detected.

In order to model the actual geometry of the source polarization used in the experiment, a numerical technique based on the Monte Carlo wave function method was used. In this method, the photodetection process is simulated so that the interference pattern builds up gradually from a series of detections at various angles. This qualitatively reproduces the experimental results and also allowed us to consider dipole-dipole interactions which become important when the atomic separation is reduced.

To more closely simulate the experimental configuration, classical motion of the ions was included in the simulation. This reduces the visibility of the interference to a value closer to the experimental result. The residual discrepancy is probably due to the approximation of modeling the ions as four-level atoms and experimental factors such as the presence of stray light on the observation screen.

Finally, we demonstrated the formation of a transient interference pattern in situations where there is no steadystate pattern by resetting the atomic state to a specified initial condition after each photodetection. In this case, a which-path argument can be used to understand the visibility of the transient patterns.

\section{ACKNOWLEDGMENTS}

We would like to thank W. Itano and D. Wineland for useful discussions. This research was supported by the University of Auckland Research Committee and the Marsden Fund of the Royal Society of New Zealand.

[1] M. Jammer, The Philosophy of Quantum Mechanics (Wiley, New York, 1974), p. 121.

[2] R. Feynman, R. Leighton, and M. Sands, The Feynman Lectures on Physics (Addison-Wesley, Reading, MA, 1965), Vol. 3.

[3] Th. Richter, Opt. Commum. 80, 285 (1991)

[4] P. Kochan, H.J. Carmichael, P.R. Morrow and M.G. Raizen, Phys. Rev. Lett. 75, 45 (1995)

[5] R.G. Brewer, Phys. Rev. A, 52, 2965 (1995)

[6] U. Eichmann, J.C. Bergquist, J.J. Bollinger, J.M. Gilligan, W.M. Itano, D.J. Wineland and M.G. Raizen, Phys. Rev. Lett. 70, 2359 (1993).

[7] D. Polder and M.F.H. Schuurmans, Phys. Rev. A 14, 1468 (1976).

[8] W.M. Itano, J.C. Bergquist, R.G. Hulet and D.J. Wineland, Phys. Rev. Lett. 59, 2732 (1987).

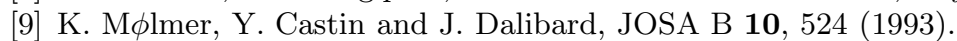

[10] T.G. Rudolph, Z. Ficek and B.J. Dalton, Phys. Rev. A 52, 636 (1995).

[11] J. Guo and J. Cooper, Phys. Rev. A 51, 3128 (1995)

[12] M.G. Raizen, J.M. Gilligan, J.C. Bergquist, W.M. Itano and D.J. Wineland, Phys. Rev. A 45, 6493 (1992)

FIG. 1. Schematic diagram of the four-level atom with decay rate $\gamma$ and detuning $\Delta$ from incident laser light of Rabi frequency $\Omega$.

FIG. 2. (a) The atomic populations versus field strength. The $\left\langle\sigma_{11}\right\rangle$ and $\left\langle\sigma_{22}\right\rangle$ curves are graphed as the solid line (they coincide) with the $\left\langle\sigma_{33}\right\rangle$ and $\left\langle\sigma_{44}\right\rangle$ curves graphed as the dashed line (again they coincide). (b) The atomic linear coherences versus field strength. The $\left\langle\sigma_{13}\right\rangle$ and $\left\langle\sigma_{42}\right\rangle$ curves are graphed as the solid line (they coincide) with the $\left\langle\sigma_{31}\right\rangle$ and $\left\langle\sigma_{24}\right\rangle$ curves graphed as the dashed line (again they coincide). Detuning is set at zero for both graphs. 
FIG. 3. Visibility plotted against field strength for three detunings. We have the axis of the polarizer aligned with the $z$ axis so that the angle $\eta$ is equal to zero. The solid line (a) is the case of zero detuning, the dashed line (b) is $\Delta=5 \gamma$ and the dashed-dotted line (c) is for $\Delta=10 \gamma$.

FIG. 4. The spherical coordinate system used in the Monte Carlo simulations with the $\epsilon_{\theta}$ and $\epsilon_{\phi}$ unit vectors defined. We also have atoms one and two at positions $\vec{r}_{1}$ and $\vec{r}_{2}$ respectively.

FIG. 5. The function $\mathcal{A}$ is shown by the solid line. The dashed line is the plot of the function $\mathcal{B}$ and the parameter $\bar{\alpha}$ is the number of wavelengths (of the incident light) separating the two atoms.

FIG. 6. Plots of intensity as a function of both angles $\theta$ and $\phi$. The incident light is linearly polarized along the $z$ axis for (a) and the $x$ axis for (b). These plots represents the light pattern distributed over the entire sphere around the two atoms.

FIG. 7. Histogram of the number of detected photons with (a) $\theta$ and (b) $\phi$ polarizations as a function of the angle $\theta$. The incident laser light was linearly polarized along the $z$ axis. The detuning was set to zero, the atomic separation was $0.5 \mu m$ and the decay rate $\gamma=5 \Omega$. The number of photons detected was 50, 086 and 49,914 for the $\theta$ and $\phi$ polarizations respectively.

FIG. 8. Histogram of the number of detected photons with (a) $\theta$ and (b) $\phi$ polarizations as a function of the angle $\theta$. The incident laser light was linearly polarized along the $x$ axis. The detuning was set to zero, the atomic separation was $0.5 \mu m$ and the decay rate $\gamma=5 \Omega$. The number of photons detected was 49,770 and 50, 230 for the $\theta$ and $\phi$ polarizations respectively.

FIG. 9. Histogram of the number of detected photons irrespective of there polarizations as a function of $\theta$ (a). Classical motion is included for (b). Both simulations used atomic separations of $3.35 \mu \mathrm{m}$ and decay rates of $\gamma=5 \Omega$.

FIG. 10. (a) The $\theta$ and (b) $\phi$ polarized light distribution versus the angle $\theta$. We have calculated 100,000 quantum trajectories in this transient simulation using the initial state $|\psi\rangle=(|1,1\rangle+|1,2\rangle+|2,1\rangle+|2,2\rangle) / 2$. There were 49,988 and $50,012 \theta$ and $\phi$ polarized photons detected respectively.

FIG. 11. The $\phi$ polarized light distribution versus the angle $\theta$ from the transient simulation using the initial state $|\psi\rangle=|1,1\rangle$. There were 50,335 $\phi$ polarized photons from the 100, 000 quantum trajectories.

FIG. 12. The $\phi$ polarized light distribution versus the angle $\theta$ from the transient simulation using the initial state $|\psi\rangle=(2|1,1\rangle+|1,2\rangle+|2,1\rangle+|2,2\rangle) / \sqrt{ } 7$. There were $50,175 \phi$ polarized photons from the 100,000 quantum trajectories. 


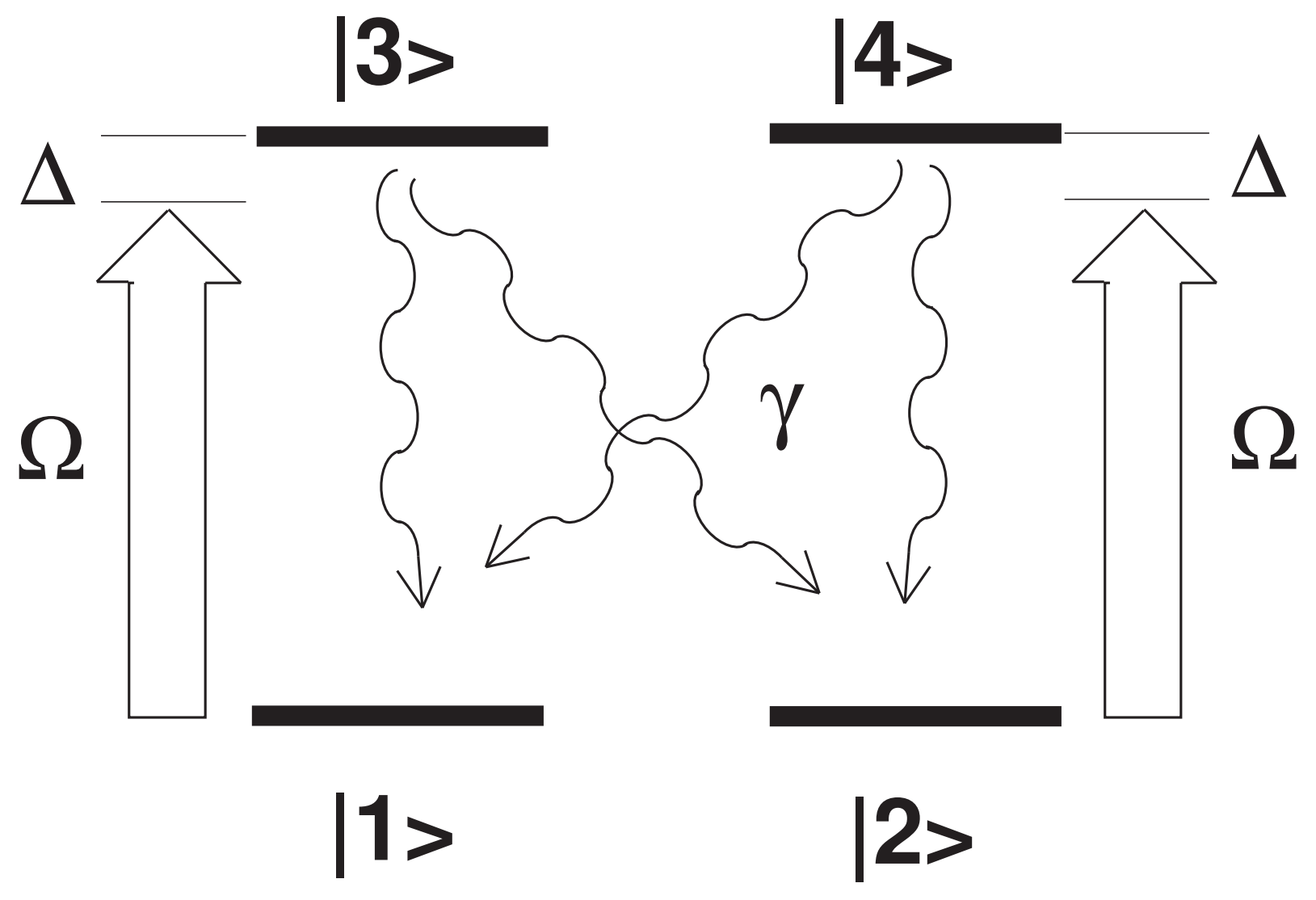

figure 1

PRA submission

T. Wong, S.M. Tan, M.J. Collett and D.F. Walls 

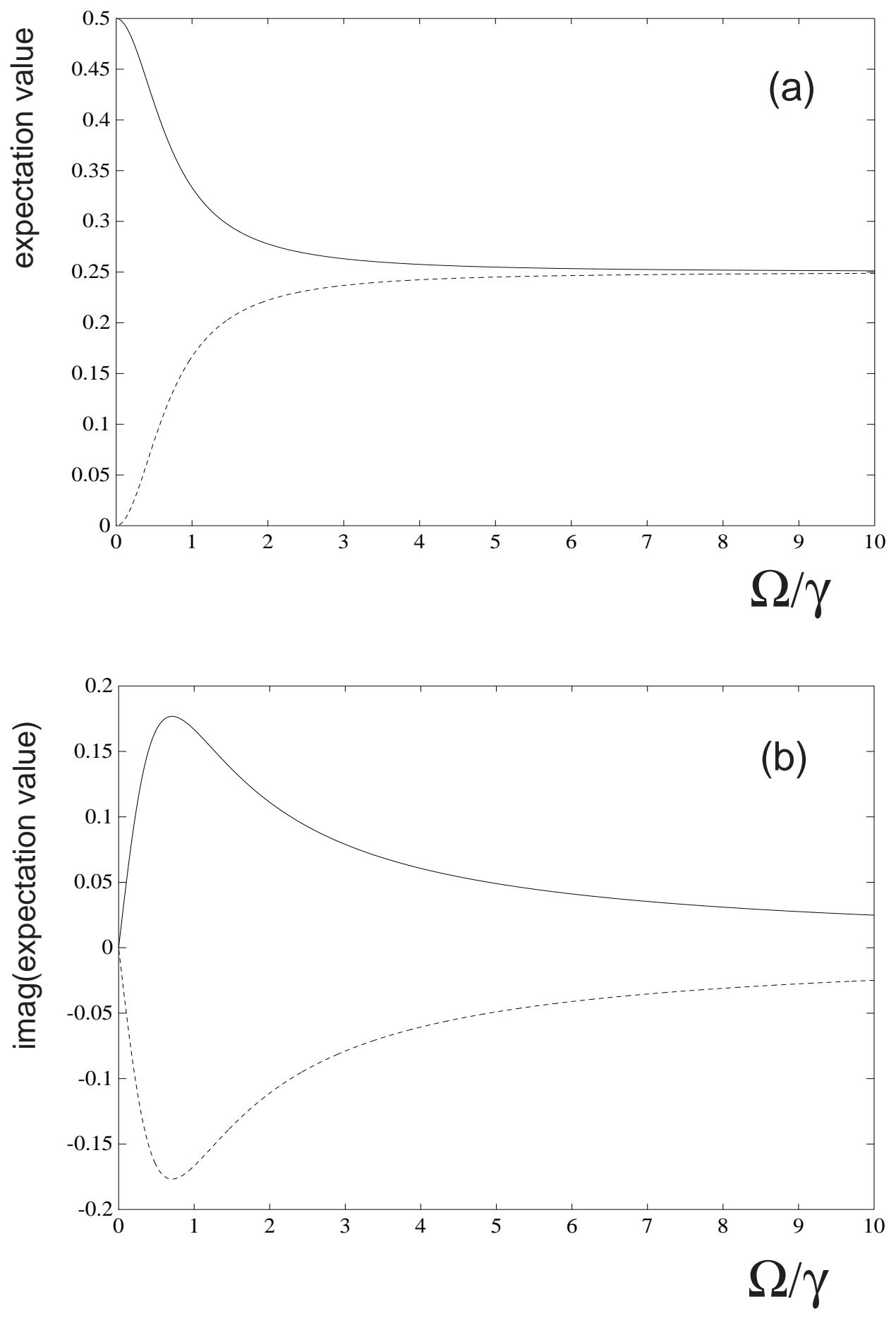

figure 2 


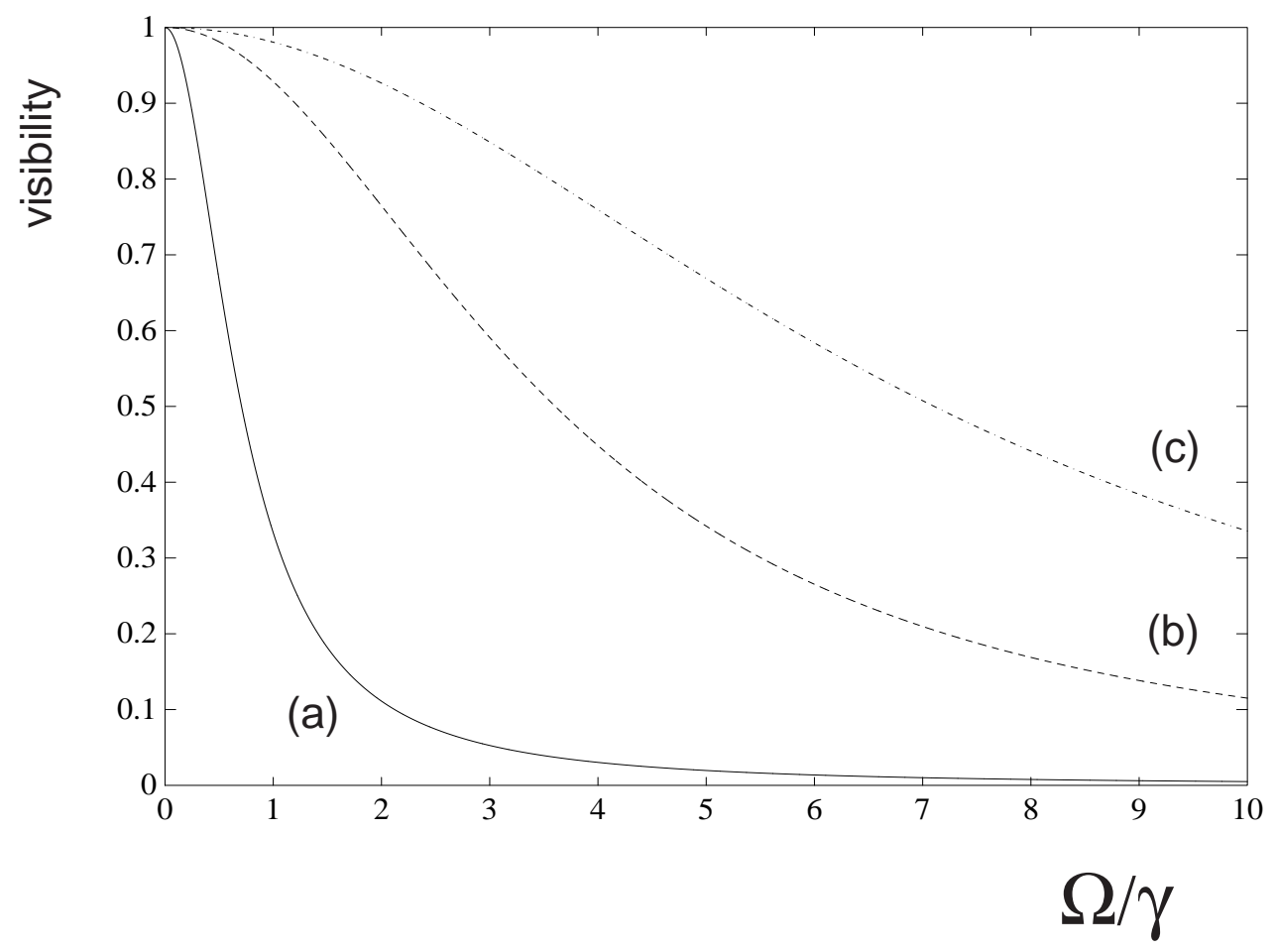

figure 3

PRA submission

T. Wong, S.M. Tan, M.J. Collett and D.F. Walls 


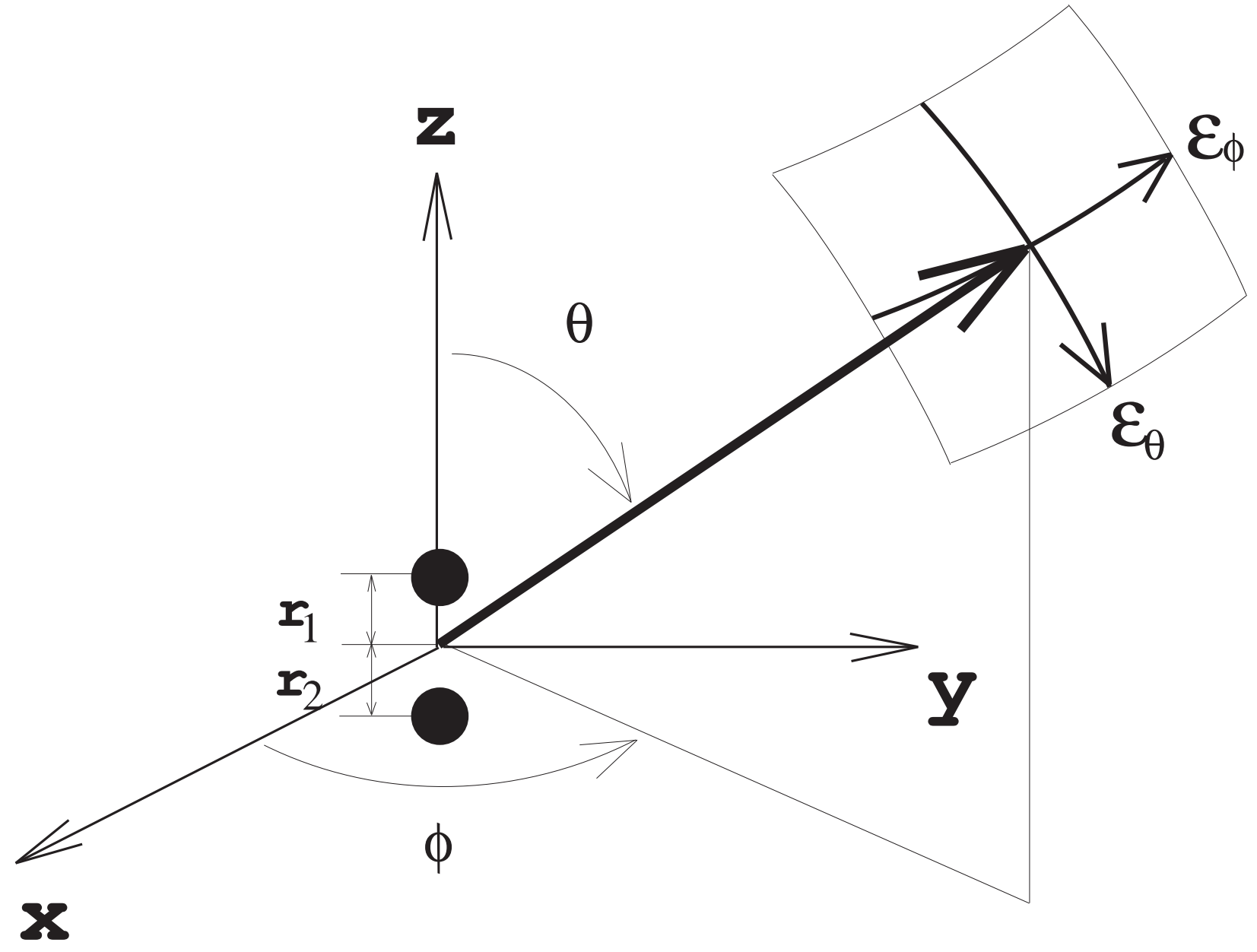

figure 4

PRA submission

T. Wong, S.M. Tan, M.J. Collett and D.F. Walls 


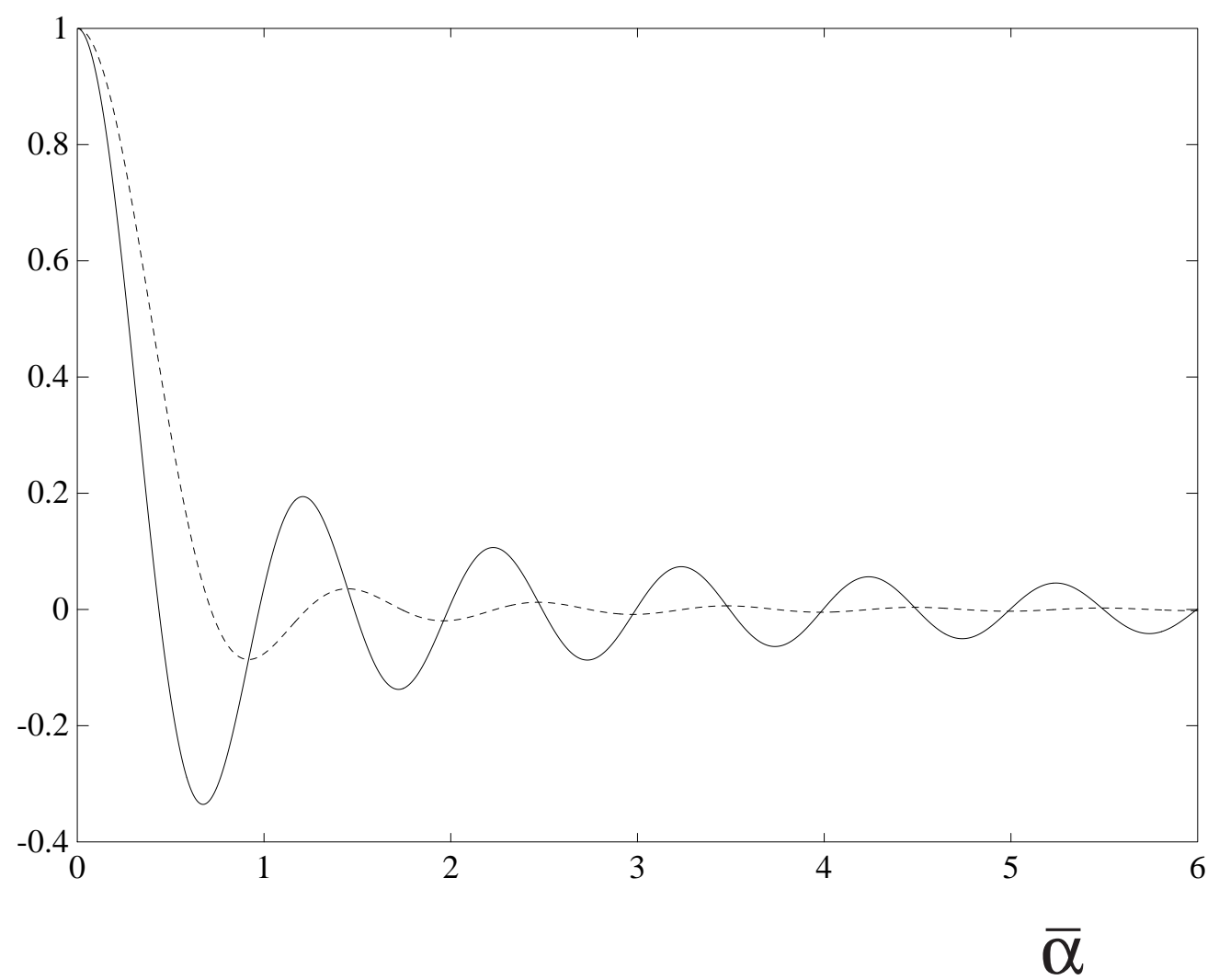

figure 5

PRA submission

T. Wong, S.M. Tan, M.J. Collett and D.F. Walls 

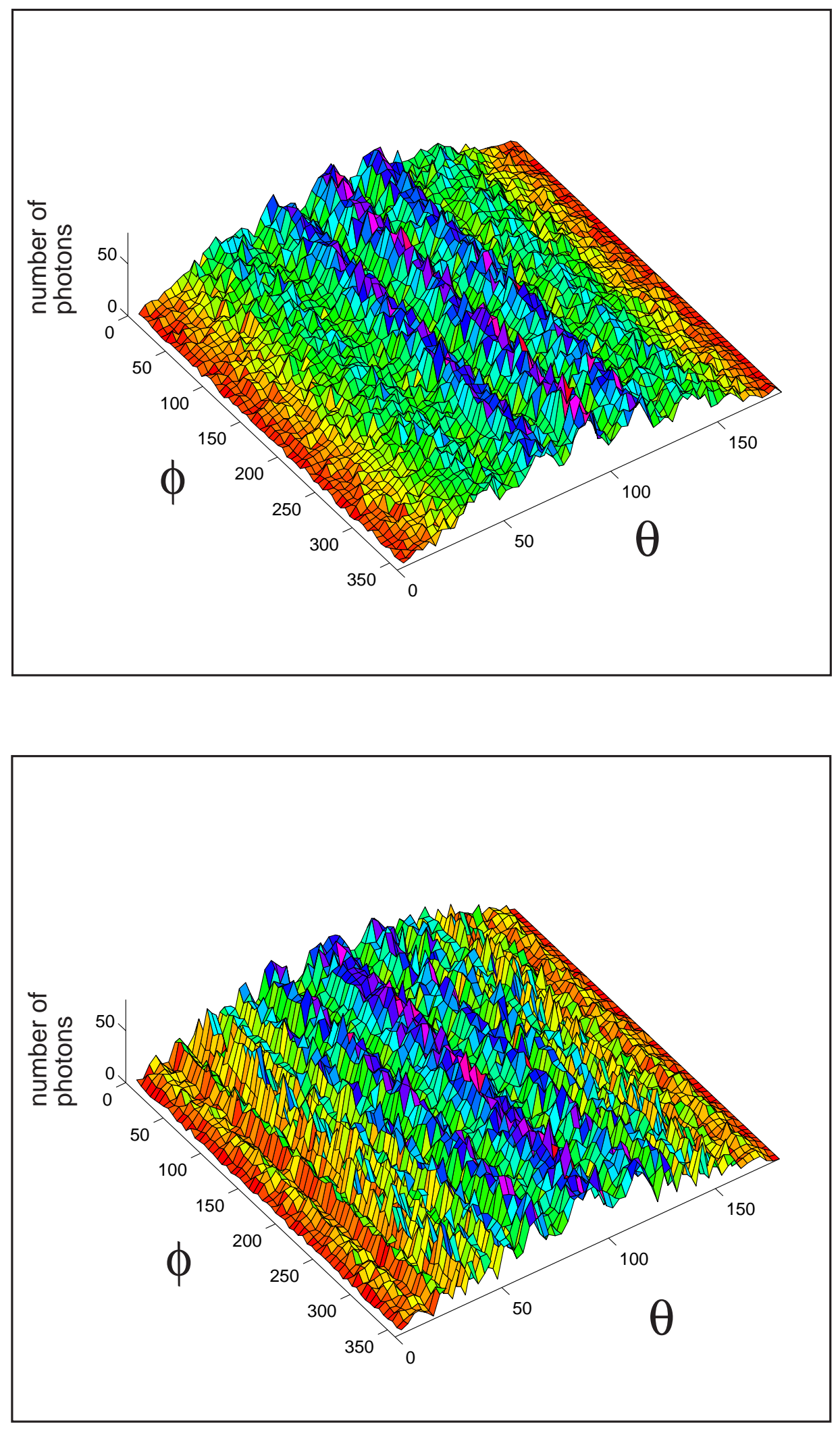

Figure 6 

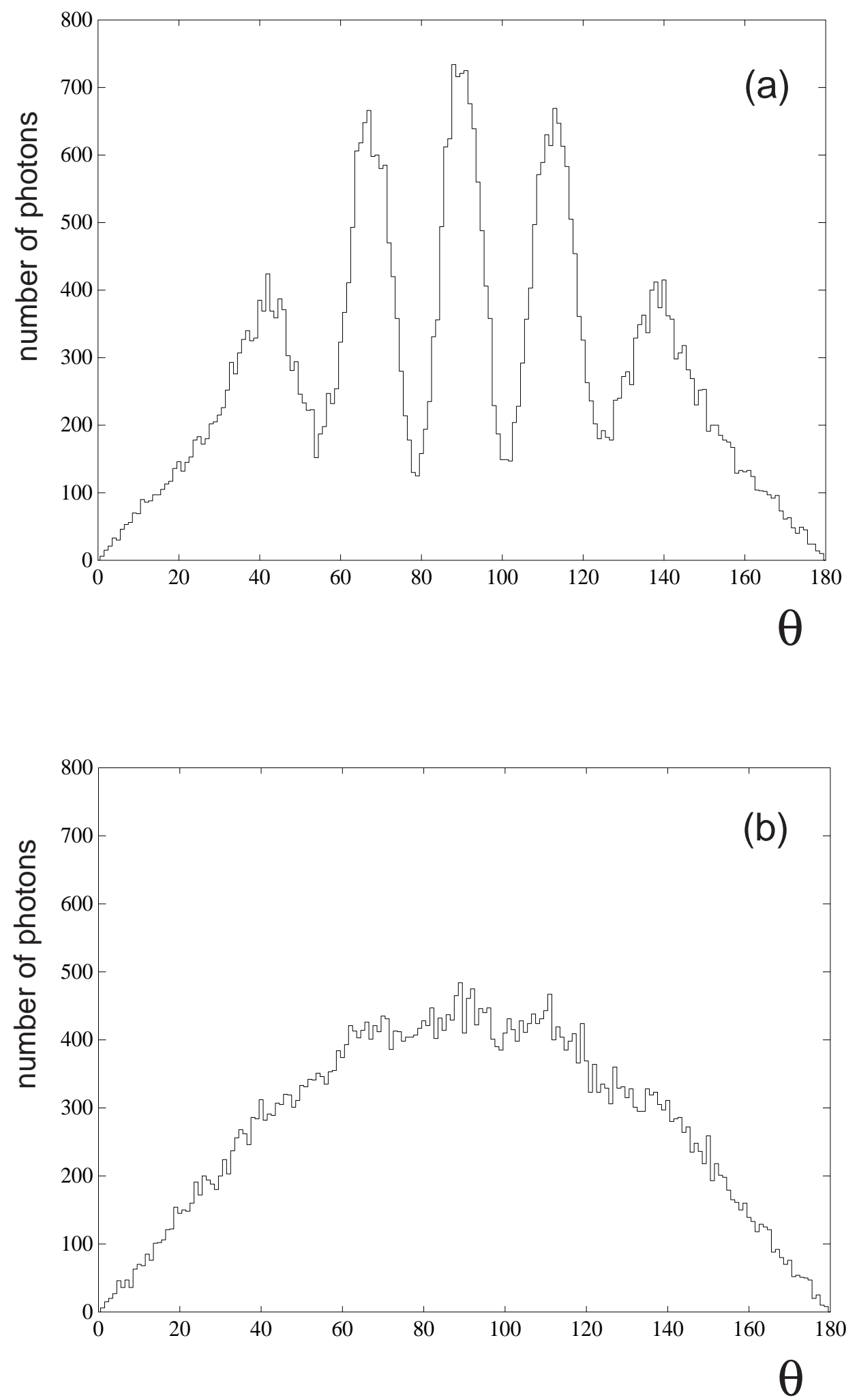

figure 7

PRA submission

T. Wong, S.M. Tan, M.J. Collett and D.F. Walls 

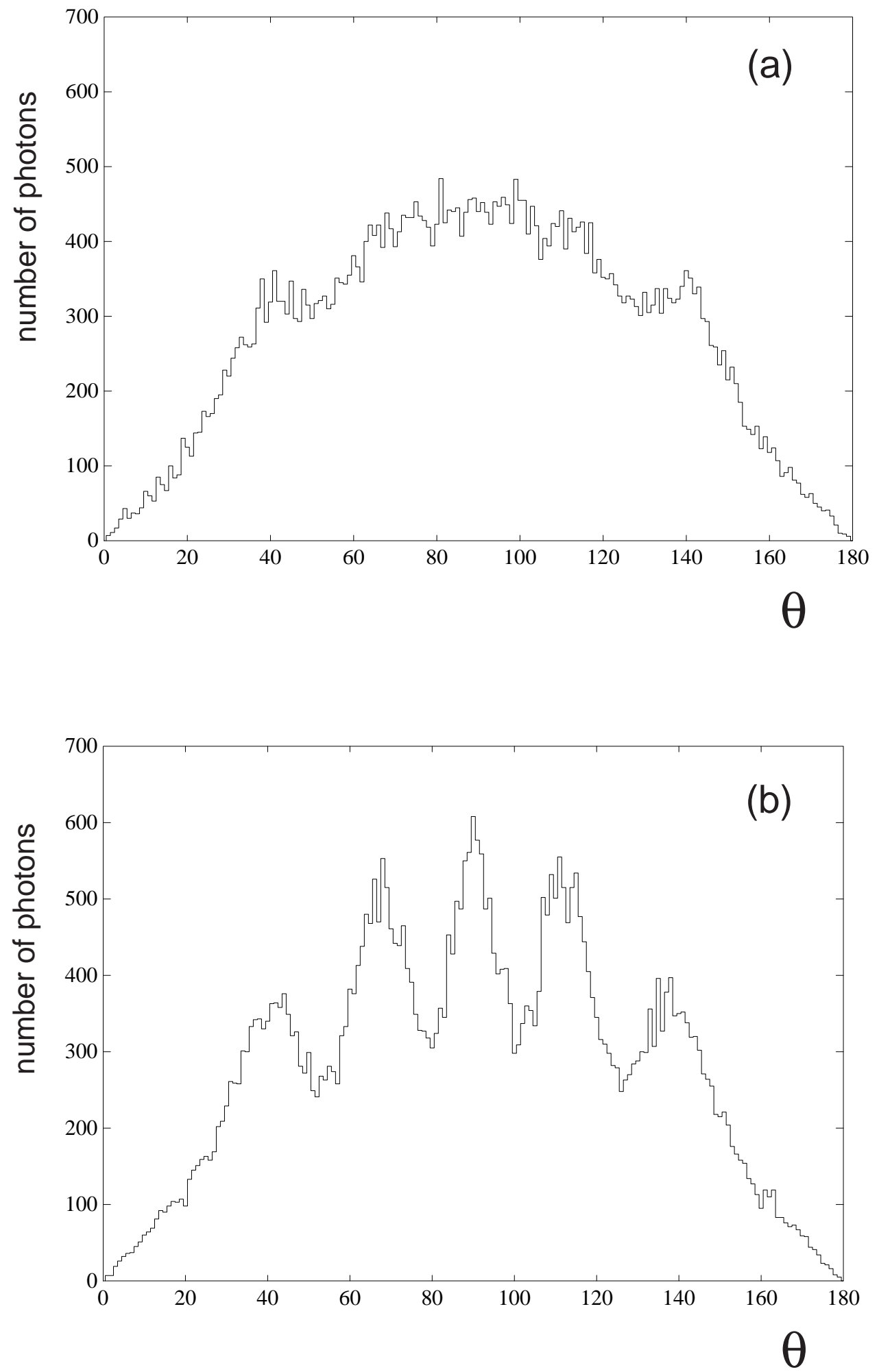

figure 8

\section{PRA submission \\ T. Wong, S.M. Tan, M.J. Collett and D.F. Walls}



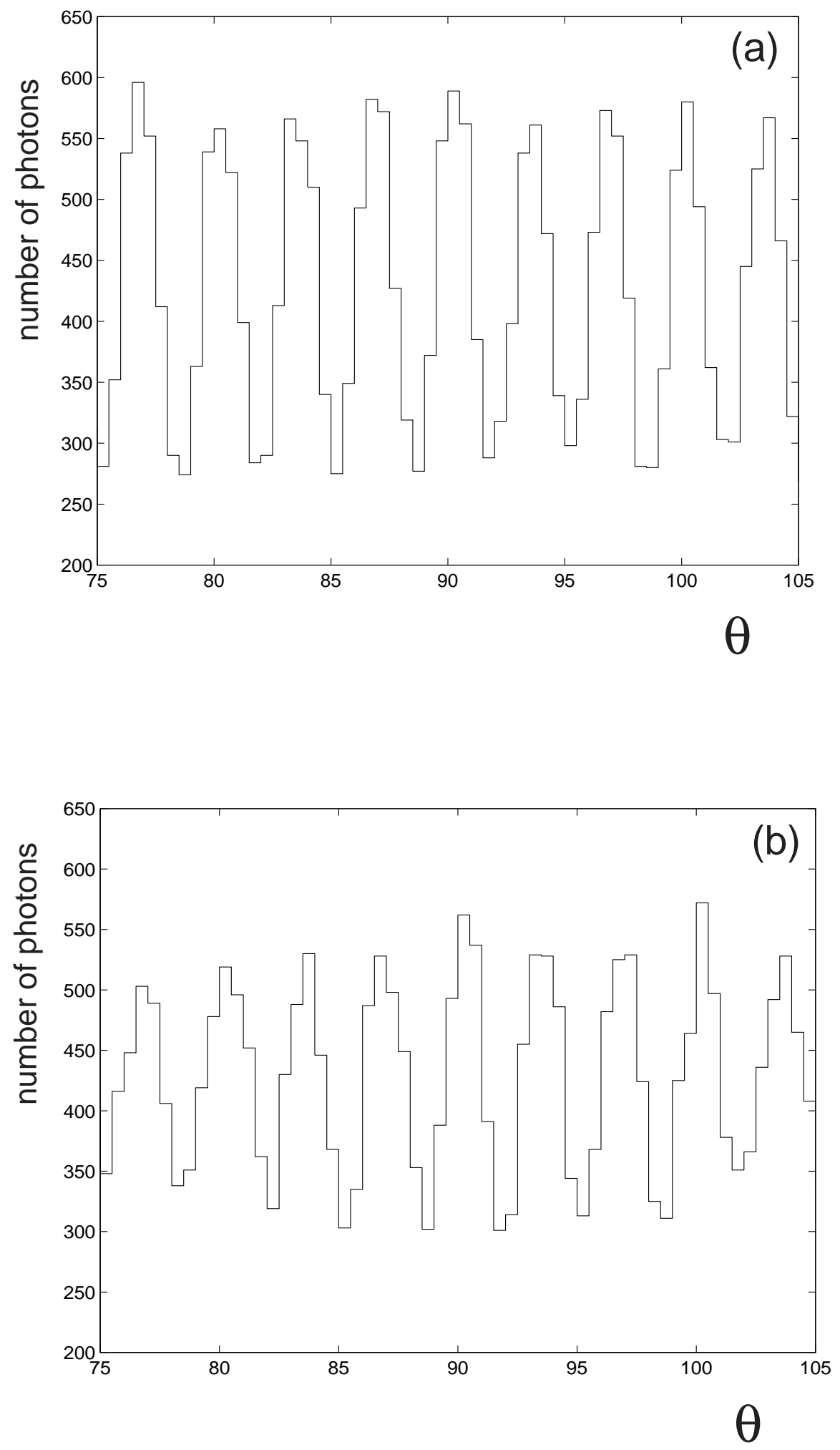

figure 9

PRA submission

T. Wong, S.M. Tan, M.J. Collett and D.F. Walls 

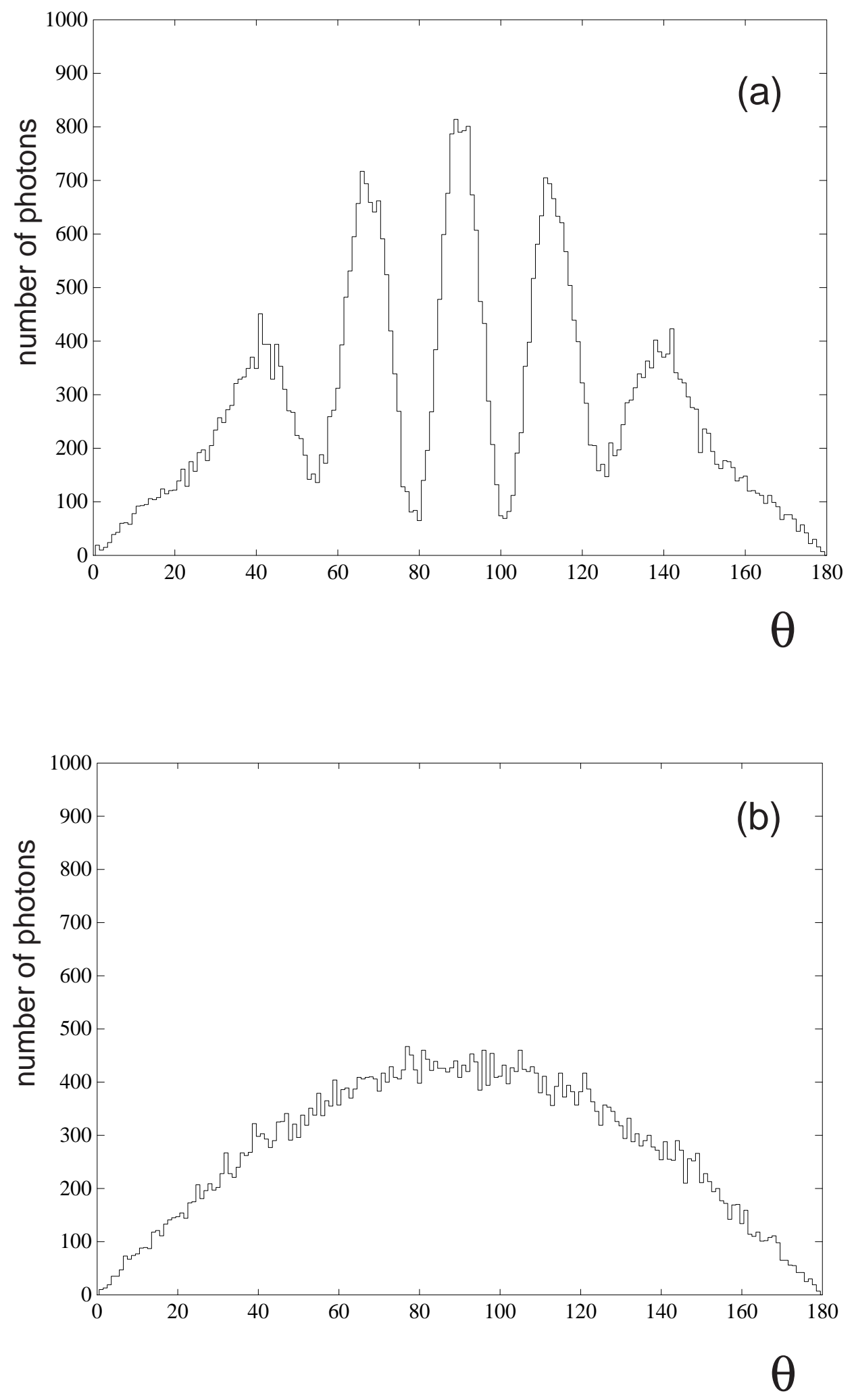

figure 10

PRA submission

T. Wong, S.M. Tan, M.J. Collett and D.F. Walls 


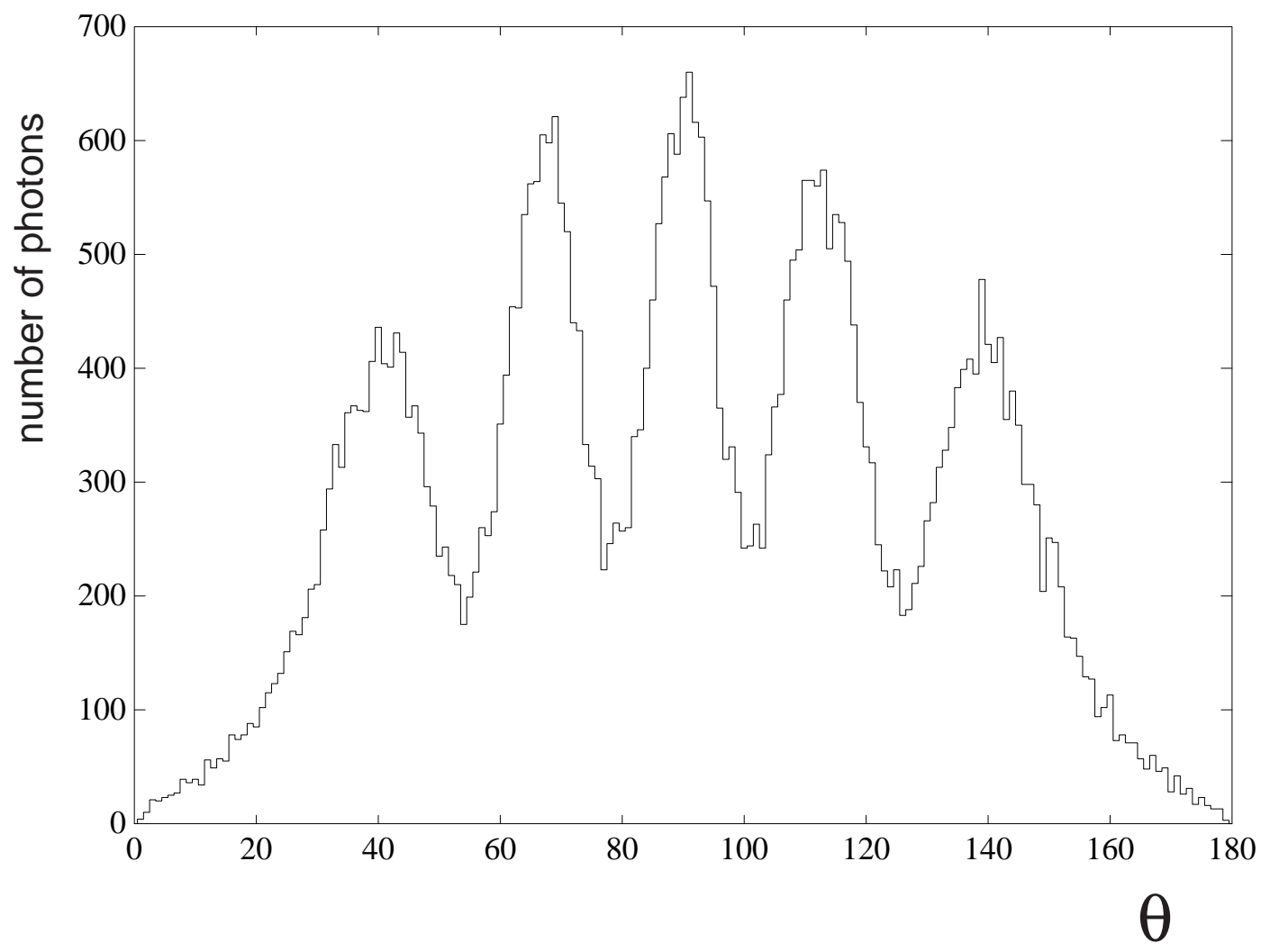

figure 11 


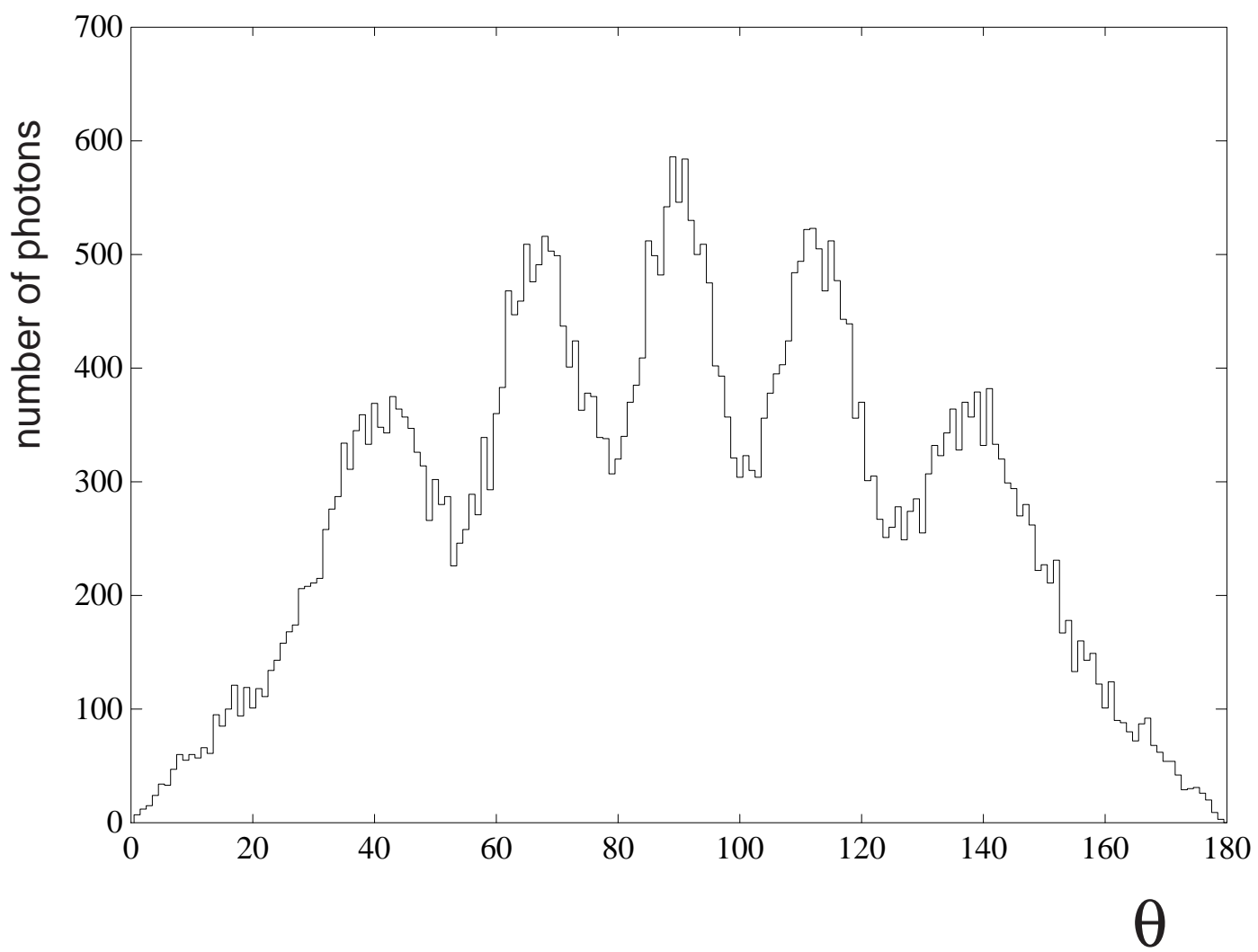

figure 12

\section{PRA submission}

T. Wong, S.M. Tan, M.J. Collett and D.F. Walls 